\title{
Racionalidades alternas en la teoría económica
}

\author{
Mara Rosas* y David Barkin**
}

\section{RESUMEN}

Las investigaciones recientes acerca del desarrollo local y las economías campesinas se realizan con una carencia de análisis teórico. La teoría económica dejó de estudiar las transformaciones que padeció un segmento importante de la población rural, los campesinos, a partir de la puesta en marcha de las políticas neoliberales porque la misma teoría de la economía campesina predijo una tendencia a la desaparición de los campesinos. Sin embargo, las preocupaciones sobre la sustentabilidad han evidenciado que las actividades económicas campesinas responden a una lógica diferente, conducente al manejo sustentable de recursos naturales; son algunos economistas neoinstitucionales quienes insisten en la necesidad de considerar que estas comunidades se conducen con una racionalidad diferente a la capitalista. El objetivo de este artículo es hacer una revisión de las diferentes corrientes teóricas que, por un lado, cuestionan la existencia de una racionalidad económica única y, por otro, aportan elementos para explicar la existencia y la dinámica de las racionalidades alternas.

Palabras clave: racionalidad alterna, actividades campesinas, sustentabilidad, economía ecológica.

Clasificación JEL: Q000.

\begin{abstract}
Since the period when neoliberal policies were implemented, economists no longer focused on social and productive processes of a large segment of peasants. Recent research on local development and rural economies lacks a theoretical framework. Much economic theory adopts some predictions of the theory of the peasant economy that predicates the disappearance of peasants. However, recent studies about sustainability evidence many productive projects structured around techniques for the sustainable management of natural resources; as a result, neoinstitutional economists are reconsidering the logic of economic activities by peasants. This contribution suggests the need for considering that peasant societies organize their activities on the basis of non capitalist rationalities; we review various theoretical currents that are questioning the existence of a single economic rationality, and examine the factors that contribute to explain the existence and dynamics of alternative rationalities.
\end{abstract}

Key word: alternate rationality, peasant activities, sustainability, ecological economics Clasification JEL: Q000.

* Profesora-investigadora del Centro de Investigación Interdisciplinaria de Desarrollo Integral Rural, Unidad Oaxaca, Instituto Politécnico Nacional. Correo electrónico mrb_ec@yahoo.com.mx. ** Profesor-investigador de la Universidad Autónoma Metropolitana, Unidad Xochimilco. 


\section{INTRODUCCIÓN}

La teoría económica ha subvalorado las formas socioeconómicas campesinas a lo largo de la historia de la industrialización en los países latinoamericanos. Hoy, el desarrollo rural todavía depende del tránsito de la economía campesina, caracterizada por la producción agrícola intensiva en mano de obra y baja productividad, a una economía que se rige por las condiciones de competencia del mercado capitalista. Sin embargo, actualmente nos encontramos en un contexto socioeconómico y ecológico diferente: los discursos sobre desarrollo sustentable y cambio climático nos están obligando a voltear hacia comunidades rurales que han reconfigurado sus estrategias económicas, aprovechando sus herencias milenarias para implantar estrategias conducentes al manejo sustentable de recursos naturales.

La construcción de opciones económicas innovadoras en un gran número de comunidades rurales combina elementos como la organización comunitaria, el conocimiento tradicional, el manejo sustentable de recursos naturales y la gobernabilidad (Rodríguez Herrera y Alvarado Ugarte, 2008; Bray y Merino, 2004; Barkin, 2004; Borrini-Feyerabend, et al., 2004; Gomero y Velásquez, 2003; Hillard et al., 2006; López, 2007; Mantilla, 2005; Murga, 2003; Reijntjes, 2009). Estos elementos corresponden a formas socioeconómicas que son propias del sistema capitalista y que hacen que los proyectos productivos que se gestionan al interior de muchas comunidades contengan los principios éticos que la economía ecológica postula como fundamentales en la construcción de una sociedad alternativa: sustentabilidad, equidad intergeneracional y justicia social.

En la teoría económica existen dos posturas acerca del tratamiento que debería darse a las actividades campesinas: la corriente ortodoxa y las posturas heterodoxas. La primera afirma que la dinámica campesina puede explicarse en el marco de la teoría neoclásica, es decir, suponiendo que los campesinos responden como maximizadores "racionales" a los incentivos del mercado. La segunda se puede dividir en las corrientes que se conocieron como campesinistas y descampesinistas, las cuales trazan sus orígenes a dos escuelas teóricas: la de Chayanov y la de Lenin, que sostienen que los campesinos poseen una racionalidad distinta a la que se presenta en el sistema capitalista. Sin embargo, la teoría de la economía campesina dejó grandes huecos teóricos, empezando porque no pudo definir a sus sujetos de estudio (Calva, 1989). Hoy la realidad campesina es todavía más compleja porque involucra el análisis del impacto del traslado de la lógica capitalista al medio rural, la emigración, la feminización de las acti- 
vidades productivas rurales, la sustentabilidad ecológica, la relación entre conservación y régimen de propiedad, las nuevas vinculaciones entre sector urbano y rural, etcétera, fenómenos que marcan cambios significativos en el quehacer campesino.

Dentro de las perspectivas heterodoxas (la teoría neoinstitucional: Ostrom, 2000; experimental: Henrich et al., 2004; ecológica: Martínez Alier y Schlüpmann, 1991; Leff, 2004; Toledo, 1992, Barkin, 1998, 2004 y 2006), y la ecología política (Martínez Alier y Schlüpmann, 1991; Martínez Alier y Roca, 2001) se han elaborado algunas corrientes teóricas que responden a algunas de las transformaciones del territorio rural a partir del análisis de una relación fundamental: régimen de propiedad-racionalidad-sustentabilidad. Estas corrientes hacen alusión a que las racionalidades alternas responden a incentivos y motivaciones sociales y no únicamente a las individuales.

El objetivo de este documento es hacer una revisión de las diferentes corrientes teóricas que, por un lado, cuestionan la existencia de una racionalidad económica única y, por otro, aportan elementos para explicar la existencia y la dinámica de las racionalidades alternas.

La conclusión es que desafortunadamente no existe una teoría que explique de manera integral la dinámica y lógica de las comunidades campesinas y que nos permita analizar la relevancia que tienen las comunidades rurales en la actualidad para lograr uno de los retos fundamentales: el progreso sustentable. Sin embargo, el progreso que existe en la economía ecológica y la teoría neoinstitucional proporciona elementos para poder avanzar en dicha tarea.

\section{ECOLOGÍA POLÍTICA Y ECONOMÍA ECOLÓGICA EN LA IDENTIFICACIÓN DE RACIONALIDADES ALTERNAS}

La ecología política relaciona los movimientos sociales, originados por el acceso desigual a los recursos, al ecologismo popular que posteriormente Martínez Alier (1994) identifica con el ecologismo de los pobres. Esta corriente agrupa a miles

de personas que son constantemente marginadas de la construcción del mercado capitalista, es decir, el desarrollo económico. Hoy, como históricamente les ha sucedido a miles de comunidades, se les expropia o se les compran sus tierras a un bajo valor, obligando a los pueblos originarios a desplazarse a otros lugares y construir nuevas modalidades de sobrevivencia; esta forma de acumulación originaria, o acumulación por desposesión (Harvey, 2009), está dada actualmente por planes de construcción de infraestructura (megaproyectos), como hidroeléc- 
tricas, presas y carreteras, o por inversiones para extraer recursos naturales, como minas y plantaciones.

El ecologismo de los pobres es una corriente ecologista que, a diferencia del culto a lo silvestre y la ecoeficiencia, ve los recursos naturales como fuente de sustento humano y, por tanto, plantea una clara vinculación entre la lucha social por la vida y la gestión sustentable de los recursos naturales (Martínez Alier, 1994). En la mayoría de los países latinoamericanos encontramos una serie de movimientos sociales, principalmente indígenas, que se encuentran luchando por la defensa de sus recursos naturales y sus formas de vida. La lucha contra grandes empresas privadas y estatales nacionales e internacionales responde a una confrontación de intereses y visones de progreso. Algunos ejemplos de movimientos sociales son el del pueblo de San Salvador Atenco, contra la expansión del aeropuerto de la ciudad de México a costa de sus tierras; los de comunidades de Guerrero contra la construcción de La Parota (presa para generar hidroelectricidad); contra la construcción de una carretera en territorio de pueblos wixarritari en la sierra huichola; conflictos agrarios entre indígenas de distintos grupos y hacendados, principalmente en Mato Grosso, Brasil, ${ }^{1}$ entre muchos otros. Víctor Toledo estima que en 1991, aproximadamente entre 300 y 400 comunidades campesinas enfrentaban luchas de tipo ambientalista por medio de 30 organizaciones campesinas sólo en México (Toledo, 1992). También existen movimientos sociales organizados en otras partes del mundo, como el movimiento Narmada Bachao Andolan, en la India; el Grupo de Acción por el Biobío, en Chile; la coalición de ONG contra la represa de Bakun, en Malasia; la Federación Costarricense para la Conservación del Ambiente (Fecon); el Consejo de Pueblos Unidos por la Defensa del Río Verde, en México. En los años, recientes muchos de los movimientos para la defensa de un recurso particular se han reunido en redes nacionales o internacionales: Red Latinoamericana contra las Presas y por los Ríos, sus Comunidades y el Agua (Redlar), la Coordinación Continental del Grito de los Excluidos, COECOceiba Amigos de la Tierra Costa Rica, el Movimiento de los Pueblos Afectados por las Represas en Brasil, el Movimiento Mundial por los Bosques Tropicales y el Movimiento de Pueblos Ambientalmente Afectados ( $c f$. Carrere, 2003).

La pregunta que surge en este contexto es ¿por qué los campesinos e indígenas están más interesados en cuidar el ambiente que el resto de la población?

\footnotetext{
${ }^{1}$ Casos extraídos del periódico La Jornada (varias fechas). Datos precisos disponible en Servicios de Comunicación Intercultural, Servindi. url: http://www.servindi.org/.
} 
La economía ecológica (EE) afirma, al igual que la economía campesina y la teoría neoinstitucional, que "las familias campesinas producen bajo una forma específica de organización y con una lógica o racionalidad económica propia" (Martínez Alier y Schlüpmann, 1991, p. 34). La aportación del estudio de las unidades campesinas de Chayanov y el uso de las formalizaciones de Georgescu-Roegen en la teoría económica abrieron paso a la idea de que "existen diversas formas de organización social de la producción, con su lógica correspondiente" (Martínez Alier y Schlüpmann, 1991, p. 35). El planteamiento teórico más acabado para entender cuál es la dinámica al interior de las comunidades campesinas en la EE la hace Georgescu-Roegen (1971).

\section{ORGANIZACIÓN CAMPESINA SEGÚN GEORGESCU-ROEGEN}

Para Georgescu-Roegen (GR), la realidad campesina es racional y cuenta con reglas económicas claras. El grado de satisfacción individual y el bienestar de la comunidad depende de "una coordenada social" (Carpintero, 2006, p. 89) que incorpora diferentes criterios, además del hedonismo. GR hace una clara separación entre economía agraria y economía campesina, señalando a la primera como gobernada por imperativos del capitalismo en la búsqueda del máximo beneficio; en contraste, a la segunda la señala como un modo de producción regido por motivaciones y pautas institucionales diferentes a las capitalistas. Los dos rasgos básicos de una economía campesina son: " $a$ ) su heterogeneidad desde el punto de vista de la tipología, y b) el carácter 'orgánico', unitario, de aldea campesina" (Carpintero, 2006, p. 91). Acentúa el hecho de que no hay una comunidad campesina, sino más bien comunidades campesinas que se diferencian entre sí por sus instituciones. Considera la aldea campesina como un todo social y económicamente indivisible. Las interrogantes que trata de contestar son: ¿cuáles son las bases de la unidad de la aldea campesina tradicional?, y ¿cómo ha logrado mantener esa cualidad a lo largo de la historia?

Siguiendo la teoría evolutiva y remontándose a las comunidades primitivas, plantea el instinto de cooperación, que no es innato sino consecuencia de las condiciones de riesgo de sobrevivencia en las que la aldea continuamente se encuentra. Cooperación para la defensa de la integridad física y para garantizar el sustento, pero, según el autor, ese espíritu cooperativo funciona únicamente cuando el grupo es pequeño e incorpora dos condiciones adicionales para que subsista la condición de unidad y estabilidad en una tierra concreta: a) que haya un equilibrio entre necesidades y recursos, y $b$ ) que éstos últimos deben estar 
próximos a la aldea. La cuestión de la estabilidad tiene como base la tradición oral.

La fisiología de las comunidades campesinas se gobierna por dos principios:

1) Sólo el trabajo crea valor y, por tanto, éste debe constituir el criterio primordial en la distribución de la renta de la comunidad.

2) Existe igualdad de oportunidades para que todos trabajen, pero no igualdad de ingresos para todos. Es decir, los miembros de la aldea campesina deben tener las mismas oportunidades para obtener su sustento, pero los frutos finales obtenidos dependerán del esfuerzo e ingenio que hayan aplicado a su trabajo (Carpintero, 2006, p. 93).

Los dos principios son las bases de dos instituciones campesinas importantes: la primera, es el aprovechamiento comunal de recursos que no son consecuencia del trabajo humano y, por tanto, nadie puede apropiárselos individualmente. La segunda institución establece que sólo lo que se posee como fruto del trabajo es una propiedad inviolable. "La tierra es para ser utilizada no para ser poseída a través de la exclusión de uso por otros" (Carpintero, 2006, p. 94). La comunidad trata de mantener el segundo principio, el de igualdad, incluso con una población creciente: "Se rotura más bosque y se reparte una fracción de la tierra roturada para cada hogar que viva en la Aldea. Y la operación se repite así para cada nueva trituración, lo que garantiza el mismo trato y evita la desigualdad cuando las tierras que se roturan en diferentes años son de distinta calidad" (Carpintero, 2006, p. 94).

El criterio de la comunidad campesina es coherente, con un criterio de elección no estrictamente hedonista, lo que significa que aparte de la cantidad de bienes poseída, la satisfacción depende también de la matriz cultural en que se inserta el individuo. La diferencia entre la racionalidad capitalista aplicada a la economía agraria y la campesina tiene una repercusión enorme en la diferencia entre el campo y la ciudad:

...no reside sólo en las costumbres, sino que incluye cada acto concreto concerniente a la producción y a la distribución así como también a la justicia social. Indudablemente la base de esta diferencia es el hecho de que la naturaleza viva impone un tipo de restricción diferente al Homo agrícola que aquella que la materia inerte impone al Homo campesino. Para comenzar, no existe paralelismo entre la ley de escala de la producción en la agricultura y en la industria. 
Uno puede cultivar trigo en un tiesto o criar gallinas en un patio pequeño. Pero ningún aficionado puede construir un automóvil solamente con las herramientas de su taller. ¿Por qué entonces debe ser una gigantesca fábrica al aire libre la escala óptima para la agricultura? En segundo lugar, el papel del factor tiempo es enteramente diferente en las dos actividades. Con artefactos mecánicos podemos acortar el tiempo para tejer una pieza de tela, pero todavía no hemos podido acortar el periodo de gestación del ganado o (en grado significativo) el periodo de maduración en las plantas. Más aún, la actividad agrícola está sujeta a un ritmo invariable mientras que en la industria nosotros podemos hacer muy bien mañana lo que no hemos decidido hacer hoy. Finalmente, hay una diferencia entre los dos sectores que llega hasta las raíces de la muy discutida ley de rendimientos decrecientes (en sentido evolucionista). Para usos industriales, el hombre ha podido aprovechar una fuente de energía tras otra, desde el viento hasta el átomo, pero para la clase de energía necesaria para la vida misma, él todavía depende por completo de la fuente más "primitiva", o sea de los animales y las plantas que lo rodean. Estas breves observaciones son suficientes para puntualizar no solamente por qué la filosofía del hombre ocupado en la agricultura difiere de la del hombre urbano, sino también por qué la agricultura y la industria no pueden regirse por las mismas leyes. (Georgescu-Roegen, 1967[1960], p. 596).

El tratamiento que Georgescu-Roegen hace de la economía campesina definitivamente es novedoso en el plano estrictamente de la crítica a la teoría convencional. La necesidad de añadir criterios culturales al criterio exclusivo económico de la racionalidad para explicar la lógica campesina es una de sus principales contribuciones. El problema en términos muy generales es que considera la aldea campesina una forma ahistórica, sin cambio en el tiempo, y su análisis no permite estudiar la relación que las comunidades han desarrollado con el sistema capitalista y los efectos que en el largo plazo se tienen de esa interacción, por ejemplo, los términos de intercambio, la explotación del trabajo campesino, las luchas sociales por la apropiación de los recursos naturales, etcétera.

El resumen que hace la EE en relación con las aportaciones de Georgescu y sus propias conclusiones se traduce en la siguiente pregunta: ¿cuál es la diferencia fundamental entre la racionalidad campesina y la racionalidad capitalista hoy? Así plantea la siguiente respuesta: "Es la contradicción entre la economía del valor de uso y la economía de la ganancia, de la expansión, del crecimiento" (Martínez Alier y Schlüpmann, 1991, p. 6), contradicción que para Martínez Alier es origen del ecologismo de los pobres caracterizado por la "defensa del 
acceso comunal de los recursos naturales, contra la amenaza del mercado o del Estado. Reacción contra la degradación ambiental causada por la pobreza, el exceso de población y el intercambio desigual" (Martínez Alier y Schlüpmann, 199, p. 10). Este análisis destaca dos aspectos relevantes en la argumentación de una racionalidad diferente a la capitalista: propiedad colectiva y manejo sustentable de recursos naturales.

\section{Racionalidades asociadas con el tipo de propiedad de los recursos}

El análisis de la clásica tragedia de los comunes (Hardin, 1968) presupone que la única alternativa para no depredar los recursos naturales es modificar su propiedad a una privada o estatal. Esta presunción, que se formula bajo el individualismo metodológico, combina la racionalidad individualista y egoísta con argumentos respecto de la eficiencia y ha sido muy influyente en la teoría neoclásica, propiciando la generación de políticas ambientales basadas en la imposición de derechos de propiedad privada sobre los recursos naturales. La EE argumenta que los teóricos neoclásicos han confundido el libre acceso a un recurso y la ausencia de acuerdos para extraerlos, con el concepto de propiedad común, que implica la existencia de derechos claros de acceso y uso de un tipo de gestión institucional determinado (Capel, 2003). No obstante, la teoría neoinstitucional ha abundando mayormente en esta cuestión y demuestra que la racionalidad egoísta, tal como la presenta la teoría neoclásica, no representa más que un caso particular del comportamiento del ser humano, "la evidencia experimental reciente, aunada al considerable volumen de nuevos campos de investigación, cuestiona la teoría convencional de forma suficientemente seria como para replantear su relevancia en el análisis de los recursos de uso común y en términos más generales, para el de los dilemas sociales", (Ostrom, 2000, p. 12).

En términos generales, la teoría económica neoclásica supone que los individuos que se enfrentan a un dilema responderán de manera limitada, y las externalidades de sus respuestas crearán condiciones que los lleven a dañarse a sí mismos y a otros sin encontrar formas de cooperación entre sí para evitar el problema. El famoso juego del dilema del prisionero ejemplifica esa afirmación. El juego es no cooperativo con información completa, cuando los jugadores no pueden comunicarse. En este caso, los jugadores son pastores que alimentan a su ganado en un pastizal común, el cual tiene un límite en cuanto al número de animales que pueden pastar de manera adecuada durante una estación, siendo el límite $L$. Si existiera comunicación, los pastores dividirían la cantidad de animales 
según $L / 2$; la estrategia de deserción implica que los pastores llevan a pastar cuantos animales consideren que pueden vender con ganancias, número mayor a $L / 2$. Si ambos limitaran su pastoreo a $L / 2$ entonces obtendrían 10 unidades de ganancia; si ambos eligieran la deserción obtendrían 0 ganancias, si sólo uno limitara su pastoreo a $L / 2$ el desertor obtendría una ganancia de 11 y el otro de -1 .

\section{Gráfica 1}

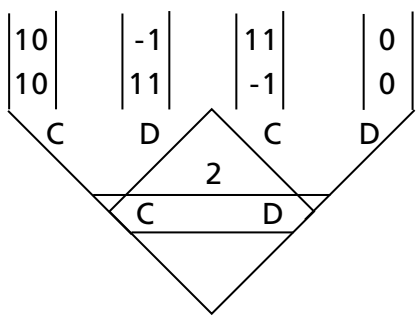

Fuente: Ostrom (2000, p. 29).

La estrategia dominante es la deserción: cuando ambos desertan obtienen una ganancia igual a cero. La opción de desertar responde a la racionalidad de los jugadores: cada uno querrá obtener más ganancia que la que permite respetar el límite del recurso. El resultado es un equilibrio del tercer mejor posible: como la estrategia dominante es desertar, elegirán esa opción ambos jugadores, quedándose con nada al fin de cuentas. Este juego ejemplifica la tragedia de los comunes de Hardin.

Los resultados de un gran número de experimentos realizados durante varios años por Ostrom (2000, p. 11) son congruentes con este resultado; cuando no hay comunicación entre los usuarios de un recurso común éstos sobreexplotan el recurso, pero cuando hay comunicación:

1) Obtienen beneficios conjuntos considerablemente mayores.

2) Cuando los pagos son relativamente bajos, la comunicación cara a cara permite a los usuarios alcanzar y mantener acuerdos cercanos a los niveles óptimos de apropiación.

3) Cuando los pagos son altos, algunos participantes están tentados a incumplir los acuerdos; los resultados conjuntos mejorados son más bajos que en la situación de pagos bajos.

4) Si se monitorea y se aplican sanciones, los usuarios están dispuestos a pagar para castigar a quienes sobreutilizan el recurso común. 
5) Cuando los usuarios discuten abiertamente y acuerdan sus propios niveles de uso y sus sistemas de sanciones, el incumplimiento de los acuerdos se mantiene muy bajo y se obtienen resultados cercanos a los óptimos.

Lo que demuestra Elinor Ostrom es que la racionalidad egoísta del individuo aislado es un caso particular de muchas otras formas de racionalidad.

\section{Economía experimental y las preferencias sociales}

La teoría de juegos también ha servido a la economía experimental para responder a la pregunta que formula esta corriente: ¿cuál es la naturaleza de las motivaciones humanas?, y ¿cómo estas motivaciones están condicionadas por las sociedades en las que la gente vive? El axioma de racionalidad económico que supone que los seres humanos se preocupan únicamente por sus propios beneficios materiales, la representación del homo economicus de la teoría económica ortodoxa, ha sido contrastado por cientos de experimentos en docenas de países. Estos experimentos han demostrado que la gente no sólo cuida sus pagos materiales (usando los términos de teoría de juegos) sino que cuida también aspectos como la justicia y la reciprocidad, aspectos que la teoría neoinstitucional llama capital social, $^{2}$ y la economía experimental preferencias sociales. Las preferencias sociales son capaces de modificar la distribución material y los costos entre grupos de personas, existen recompensas o sanciones sociales a las personas que actúan o no de manera prosocial, lo cual significa que la gente actúa en función del tipo de sociedad al que pertenece (Henrich et al., 2004).

Este conjunto de investigaciones claramente demuestra que las preferencias sociales están moduladas por el ámbito económico, social y cultural. La investigación etnográfica e histórica sugiere que las sociedades humanas, con sus propias formas de organización social e instituciones, tienen diferentes sistemas de parentesco, circunstancias ecológicas y diferentes grados de integración al mercado y muestran poca variación del comportamiento social. Los patrones de comportamiento reflejan contextos locales variados; sin embargo, la economía experimental considera que estos estudios sólo pueden ofrecer pruebas circunstanciales del comportamiento humano. Muchos modelos diferentes de acción hu-

\footnotetext{
${ }^{2}$ Un término muy controvertido que refleja la cuantificación de procesos sociales, figura rechazada por muchos estudios preocupados por los valores culturales y políticos de las formas de cooperación entre los miembros de una comunidad o del proceso de construcción de alianzas entre comunidades (Fine, 2009).
} 
mana son coherentes con los registros etnográficos e históricos; la cuestión fundamental para la economía experimental es cómo se puede asegurar que las diferencias en las motivaciones humanas derivan de las diferencias entre las sociedades; quizá la diferencia pueda radicar en las diferencias ecológicas y tecnológicas principalmente o en las circunstancias históricas.

Para esta corriente, los experimentos permiten distinguir entre los comportamientos decisivos para lograr objetivos como reputación, reciprocidad de largo plazo, conformidad con las normas sociales y los comportamientos valorados individualmente. Utilizando los juegos del ultimátum, de bienes públicos y del dictador, se utilizaron datos etnográficos para explorar los motivos que subyacen en la diversidad de la sociabilidad humana.

El juego del ultimátum es un juego de negociación sencillo. En este juego los sujetos están en pares: al primer jugador, llamado el proponente, se le asigna provisionalmente una suma de dinero: el pastel. El proponente puede ofrecer cualquier porción del pastel a una segunda persona, a menudo llamado el contestador, quien responde conociendo tanto la oferta como la cantidad total del pastel; entonces tiene la oportunidad de aceptar o rechazar la oferta del proponente. Si el contestador acepta, recibe la cantidad ofrecida y el proponente recibe el resto. Si el contestador rechaza la oferta, entonces ninguno de los jugadores recibe nada. El juego del dictador se juega exactamente como el juego estándar del ultimátum, excepto que al contestador no se le da una oportunidad de aceptar o rechazar la oferta. El proponente sólo dicta la decisión de cómo se repartirá el pastel. En el juego del dictador las ofertas positivas no pueden ser resultado del temor al rechazo. Así, cuando se usa junto con el juego del ultimátum, esta herramienta experimental permite a los investigadores determinar si los proponentes hacen ofertas positivas por un sentido de equidad o de un miedo al rechazo. Los experimentos de los bienes públicos fueron destinados a investigar cómo se comportan las personas cuando los intereses individuales y de grupo están en conflicto. Se utilizaron dos variantes: el formato de contribuciones voluntarias y los recursos comunes en forma de fondo; la única diferencia es que en el primero, los sujetos pueden contribuir al bien común y en el segundo pueden abstenerse y usar los recursos comunes para beneficio privado (Henrich et. al., 2004, p. 12).

Los resultados de los experimentos se resumen en cinco puntos: $i$ ) no hay ninguna sociedad en la que el comportamiento experimental sea coherente con el modelo canónico de libros de economía; ii) hay mucha más variación entre los grupos que la informada por el experimento; iii) las diferencias entre las sociedades en la integración del mercado y la importancia de las cooperaciones 
explican una porción considerable de la variación del comportamiento entre los grupos; iv) en lo individual, las variables económicas y demográficas no explican el comportamiento dentro o entre grupos, y $v$ ) los juegos experimentales a menudo reflejan los patrones de interacción en la vida cotidiana (Henrich et al., 2004, p. 10).

\section{Actividades campesinas y manejo sustentable de recursos naturales}

La racionalidad que subyace al manejo sustentable de recursos naturales es una cuestión que tiene que ver fundamentalmente con las actividades económicas campesinas. En términos de conservación de la biodiversidad agrícola, silvestre y uso energético, la economía campesina tradicional es superior a la capitalista. "Las variedades de plantas agrícolas domesticadas (como también las razas de animales domésticos) fueron creadas a partir de las silvestres por el trabajo y el ingenio de generaciones de agricultores [y ganaderos]" (Martínez Alier, 1992, p. 133). Fueron creadas con objetivos muy diferentes a los de la agricultura moderna, mientras que las modificaciones genéticas recientes fueron diseñadas con el fin de incrementar la producción por hectárea y las innovaciones en cuanto a los procesos de cosecha y recolección requieren un alto consumo de energía de combustibles fósiles. "El libro de Rachel Carson de 1962 contra los pesticidas químicos, los estudios del flujo de energía en la agricultura por D. Pimentel en 1973, G. Leach en 1975 y otros autores, como Naredo y Campos en 1980 quienes, a partir de una sugerencia de Howard Odum, mostraron que la agricultura moderna era inferior en cuanto a su eficiencia energética" (Martínez Alier, 1992, p. 136).

El objetivo final de la agricultura moderna ha sido el crecimiento de la producción que, según la economía ortodoxa, eliminará la pobreza y ésta, a su vez, la degradación; esta perspectiva afirma que los pobres son quienes contaminan y causan degradación ecológica. Pero la EE muestra evidencia de que, en primer lugar, el crecimiento per se no genera una distribución equitativa de los recursos, lo que significa que no reduce la pobreza; es más, los trabajos de Kaldor y Kuznets ayudan a identificar el trade-off entre reducir la desigualdad y el impulso del crecimiento (Fisher y Erickson, 2007, p. 53). Además, contrario a la afirmación de la teoría neoclásica de que los pobres son los que más contaminan, los ricos, al hacer un uso más intensivo de energía (con el uso de automóviles, aviones y bienes suntuarios), generan una degradación creciente.

La EE pone en evidencia la incapacidad de la teoría ortodoxa de resolver problemas tan urgentes como el desempleo, la pobreza y la degradación ambien- 
tal. La justicia social (en el presente y para el futuro), la equidad distributiva y la sustentabilidad son sus tres ejes de análisis. No obstante, después de su construcción teórica de base, fundada en los principios de la termodinámica y la dinámica de la económica abierta, la EE ha avanzado muy poco en lo que Herman Daly considera como un objetivo esencial de la EE: la redefinición del camino al progreso (Erickson y Gowdy, 2007). El resultado es una apuesta al regreso de políticas macroeconómicas fincadas en principios keynesianos y la reorientación de las políticas económicas hacia fines sociales, económicos y ecológicos e incluso una visión muy pesimista del futuro cercano.

Aun cuando la EE reconoce que las técnicas agrícolas tradicionales son ecológicamente mejores que las capitalistas, se plantea serias dudas acerca de su alcance: “¿Cómo se protegerían contra la inmigración las pequeñas ecotopías? Los movimientos ecologistas populares ¿contribuirán notablemente con sus acciones a la internalización de externalidades? ¿En qué escuelas se enseña a los campesinos tradicionales que ellos son, posiblemente, baluartes de la ecología contra el sistema de mercado generalizado y contra la modernización tecnológica?" (Martínez Alier, 1992, p. 83).

Estas preguntas no son ingenuas. Toman en cuenta las políticas comerciales de orden internacional que privilegian a las empresas trasnacionales con sus técnicas y formas de apropiación de semillas nativas, la innovación tecnológica que no respeta el principio de precaución, el discurso de que con el crecimiento se acaba la desigualdad y el predominio de la visión neoclásica que presupone que con la asignación de valores económicos la degradación ambiental desaparecerá. Pero soslayan aspectos importantes, como el hecho de que así como el sistema capitalista se encuentra en constante cambio, la agricultura tradicional no ha permanecido inerte; la lucha social campesina e indígena ha tenido sus triunfos: "lo que más impresiona, sin embargo, son los altos niveles de organización y de éxito productivo y social alcanzados por las más avanzadas de las organizaciones campesinas" (Toledo, 1992, p. 39). No sólo buscan conservar recursos para uso común -como lo afirma el ecologismo popular-, sino que buscan la autonomía en su sentido más amplio: social, político y económico. Entonces, como lo afirma Toledo, si la producción campesina se caracteriza por un predominio relativo del valor de uso sobre el valor de cambio, entonces "debe de contar con un conjunto de estrategias, tecnologías, percepciones y conocimientos que hacen posible la reproducción social sin menoscabo de la renovabilidad de los recursos naturales (ecosistemas)” (Toledo, 1992, p. 41). ¿Cuáles son esas estrategias? Es algo en lo que la EE no profundiza y mucho menos plantea la posi- 
bilidad de que la sustentabilidad y la generación de excedentes puedan coexistir en organización social alguna.

\section{RACIONALIDAD EN LA TEORÍA DE LA ECONOMÍA CAMPESINA}

No existe una teoría general de la economía campesina. La indagación teórica en los cuarenta años pasados se enfrascó en discusiones acerca de cómo debería estudiarse al campesinado: ¿utilizando las categorías y leyes de la economía política clásica o con la teoría neoclásica? o si "los campesinos integran un tipo específico de economía cuya organización interna y leyes fundamentales de operación, esencialmente invariables en todas las épocas históricas, deben analizarse y formularse como un sistema económico especial o modo de producción campesino" (Calva, 1988, p. 9). En América Latina, el segundo postulado tuvo mayor fuerza; la indagación teórica, por tanto, se centró en la investigación acerca de la naturaleza del campesinado, su definición como clase social, sus formas de organización autonómica, sus formas de vinculación con el sistema dominante y su futuro (Cortés y Cuéllar, 1986)

En el nivel de la implantación de política pública, el problema que se plantearon gobiernos y organismos internacionales fue acerca de la función del campesinado en el desarrollo económico. Optaron por un concepto de campesino que no distaba del de un empresario con la dotación de condiciones materiales propias de los campesinos: cantidad de tierra, técnicas y algunos rasgos de gestión empresarial que en el plano teórico hacían referencia a que no existía ninguna característica particular entre las motivaciones de un empresario y las de un campesino. Los resultados de esas políticas, a finales del siglo pasado, fue la presencia de empresas agrícolas medianas, a costa de un deterioro en la situación de los campesinos. La pregunta que refiere Gustavo Esteva (citado por Cortés y Cuéllar, 1986, p. 64) es: ¿qué ocurre realmente con este grupo de personas a las que no podemos estudiar con precisión y a las que no podemos definir, y que actualmente son las dos terceras partes de la humanidad y casi la mitad de quienes habitan este país?

A principios de los años setenta, la discusión teórica se centró en el devenir de los campesinos, planteándose las famosas discusiones entre campesinistas contra descampesinistas, con sus respectivos fundamentos teóricos. La primera era guiada por las aportaciones de A. Chayanov, incluido dentro de la corriente formalista que deriva de la escuela marginalista austriaca, pero también relacionado con la corriente de antropología económica en la que se ubican Sha- 
nin, Wolf y Thorner, entre otros (Calva, 1988). La segunda se informaba de Lenin, ubicado en la perspectiva marxista, que fundamentalmente discute la naturaleza del campesinado y su definición como clase social.

Dentro del ámbito de la sociología marxista, que se convertía en el paradigma dominante de la región, una primera respuesta consistió en aplicar directamente las categorías del materialismo histórico y tratar de definir la situación de clase del campesinado a partir de su relación con los medios de producción. Pero para el marxismo clásico la identificación social del campesinado había sido un problema de imposible solución y, en el mejor de los casos, el tratamiento acababa viéndolo como una clase en transición. Así se leyó a Lenin desde una óptica que destacaba las vías de desarrollo del capitalismo en el campo (Cortés y Cuéllar, 1986, p. 67) .

La contradicción subyacente entre la postura de Chayanov y la de Lenin se puede entender tanto por el modelo teórico y metodológico empleado como por la situación histórica. Ambos se refieren a tipos sociales que tienen motivaciones o lógicas diferentes: para Lenin es decisivo mostrar que existe una transición de sistema productiva, de la producción campesina a la producción capitalista, mientras que Chayanov demuestra que la estructura social de la unidad económica campesina (UEC) no es capitalista, por tanto, refiere a una lógica o una racionalidad particular.

Las aportaciones de Alexander Chayanov llegan en un momento decisivo para Rusia. La transición del sistema zarista al sistema socialista, sin la aparición intermedia del capitalismo, explica parte de los fracasos del modelo ruso. Chayanov formaba parte de un ala política conocida como neopopulista, es decir, intelectuales procampesinistas que apoyaban una transición económica sustentada en la actividad económica campesina.

Su análisis de las UEC consiste en una investigación en el marco de la “escuela para el análisis de la organización y producción campesinas". Esta corriente parte del supuesto de que la economía campesina "no es típicamente capitalista, en tanto no se pueden determinar objetivamente los costos de producción por ausencia de la categoría salarios. De esta manera, el retorno que obtiene un campesino luego de finalizado el año económico no puede ser conceptuado como parte de algo que los empresarios capitalistas llaman 'ganancia'" (Chayanov, 1985, p. 8). 
El objetivo de Chayanov es descubrir las leyes que gobiernan la producción del sujeto de la UEC: la familia. La intensidad de la actividad de la fuerza de trabajo y el volumen de producción dependen fundamentalmente de la composición (cantidad de miembros en posibilidad de trabajar) y el tamaño de la familia. El tamaño de la familia no es el único factor determinante del nivel de producción de la unidad de explotación, pero de éste dependen sus límites máximo y mínimo. El máximo se determina por la mayor cantidad de fuerza de trabajo disponible y el más bajo por los medios mínimos de subsistencia de la familia. Sin embargo, las variaciones del nivel de ingreso de la UEC respecto de esos dos límites depende adicionalmente de:

...hasta qué punto son útiles estos trabajadores: qué parte del tiempo potencial de trabajo se utiliza realmente; cuál es la intensidad de su trabajo o el grado de autoexplotación: con qué medios técnicos de producción ingresa la fuerza de trabajo al proceso de producción; qué nivel de productividad logrará como resultado final esta fuerza de trabajo de acuerdo con las condiciones naturales y la situación de mercado (Chayanov, 1985, p. 68).

La diferencia en el ingreso neto de cada UEC depende de dos factores que determinan su productividad anual: $i$ ) el grado de intensidad del trabajo desplegado dentro de la UEC (gasto de energía que el trabajador quiere gastar a lo largo del año), y ii) las condiciones técnicas y económicas. El trabajo más intenso en tierras poco fértiles, aunado a una situación desfavorable en el mercado para su producción, no generará un mayor ingreso. El grado de intensidad del desgaste físico es la medida de la autoexplotación de la fuerza de trabajo campesina.

Los factores que determinan el nivel de intensidad del trabajo son:

1) Estructura interna de la familia.

2) Satisfacción de las necesidades de consumo de la familia.

3) Condiciones de producción que determinan la productividad de la fuerza de trabajo.

La presión que genera la satisfacción de necesidades de consumo es medida con un coeficiente que relaciona la cantidad de consumidores respecto de la cantidad de trabajadores. La medida de la autoexplotación depende en mayor grado del peso que ejercen las necesidades de consumo de la familia. A mayor necesidad de consumo corresponde una mayor intensidad del trabajo y mayor 
autoexplotación. Por lo que el ingreso está en función de las necesidades de consumo y de las condiciones en las que se realiza el trabajo (gráfica 2). Por condiciones de producción se entiende la disponibilidad de medios de producción, cantidad y calidad de la tierra a la que se aplica la fuerza de trabajo. La UEC produce hasta el punto en que se iguala la intensidad del trabajo anual con el grado de satisfacción de necesidades.

\section{Gráfica 2}
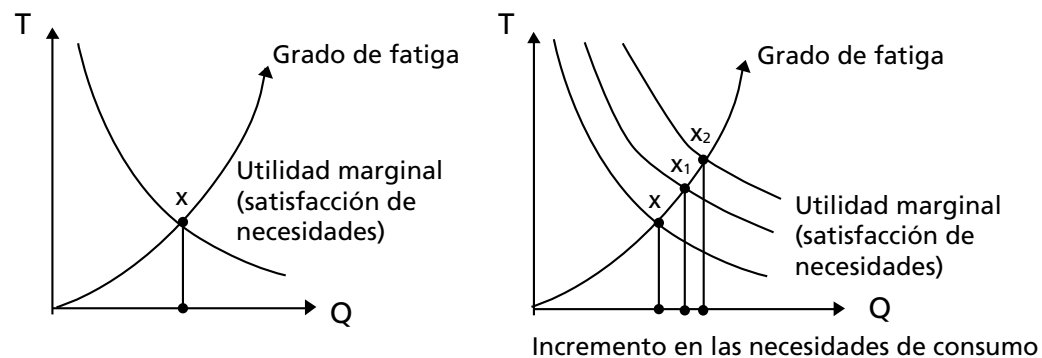

Fuente: Elaboración propia a partir de Chayanov (1985).

El objetivo de la producción de la UEC es la satisfacción de necesidades, no la maximización de la tasa de ganancia. Cuanto más duro es el trabajo, comparado con la remuneración, más bajo es el nivel de bienestar en el cual la familia campesina deja de trabajar. En la gráfica 2 se muestra que el grado de autoexplotación de la fuerza de trabajo se establece por la relación entre la medida de la satisfacción de las necesidades (medido por el valor de la producción $Q$ ) y la del peso del trabajo $(T)$.

El grado de fatiga que responde a la adquisición de un peso adicional es más que proporcional al esfuerzo que hace el trabajador por adquirirlo. La evaluación subjetiva que hace la familia del vigésimo o trigésimo peso resulta excesivamente alto, puesto que la familia que dispone sólo de estas sumas en el año sólo podrá cubrir las necesidades que considera más importantes. En el punto de equilibrio $x$, según la gráfica 2 , la evaluación subjetiva del peso obtenido por el trabajo marginal se iguala con la evaluación subjetiva de las fatigas de este trabajo. Respecto de la utilidad marginal, cada peso subsiguiente al equilibrio se evaluará por debajo de las fatigas para obtenerlo. De este análisis se deriva una pregunta importante: ¿asumiendo esta lógica de producción, existen ganancias derivadas del nivel de autoexplotación de la UEC? La respuesta es negativa. Si el beneficio neto $(\mathrm{BN})$ es igual a: 


$$
I B+G M+S=B N
$$

donde $I B$ es ingreso bruto, $G M$ gasto en maquinaria y materias primas, y $S$ salarios. Chayanov deduce que a diferencia del sistema capitalista, en el que todos estos elementos pueden expresarse en una misma unidad, en las UEC no existe la categoría de salarios. El consumo de la fuerza de trabajo se expresa en unidades físicas, la remuneración de los miembros de la UEC se representa como un excedente, la diferencia entre $I B$ y $G M$ deriva en una evaluación subjetiva que es satisfactoria o insuficiente según las fatigas $(F)$ que implicó el trabajo:

$$
\begin{gathered}
(I B-G M)>F \text { y }(I B-G M)<F \\
\frac{(I B-G M)}{F} \underset{\leq}{\geq} X
\end{gathered}
$$

Donde $X$ será la evaluación subjetiva de la fatiga de un día de trabajo. Frecuentemente, el acercamiento al equilibrio básico interno de la UEC hace que sean aceptables remuneraciones muy bajas por unidad doméstica, lo cual le permite existir en condiciones que llevarían a la ruina a las empresas capitalistas.

Respecto de la organización de la UEC, Chayanov considera que es posible determinar la relación técnica más apta entre factores de producción para determinar el tamaño adecuado de la UEC, reducir al máximo sus costos de producción y lograr los más altos ingresos.

Otra gran diferencia que ve Chayanov respecto de la organización de cualquier otro sistema de producción es que la fuerza de trabajo es un factor fijo (gráfica 3), porque depende de la composición familiar, no puede aumentarse o disminuirse a voluntad. Así, los demás medios productivos se ordenarán alrededor de la fuerza de trabajo y la tierra. Si la tierra es poca respecto del número de miembros de la familia, se reduce considerablemente la actividad agrícola y la mano de obra familiar incursiona temporalmente en otras actividades no agrícolas, comerciales o artesanales.

La carencia de tierra no es la única razón que ubica Chayanov para explicar que algunos miembros de la UEC se dediquen a actividades no agrícolas; también influye la distribución del tiempo del trabajo agrícola. Existen periodos del año en los que las unidades domésticas se encuentran inactivas y otros en los que hay una carga intensa de trabajo. En general, Chayanov plantea que la divi- 
Gráfica 3. Composición técnica e ingreso de la UEC

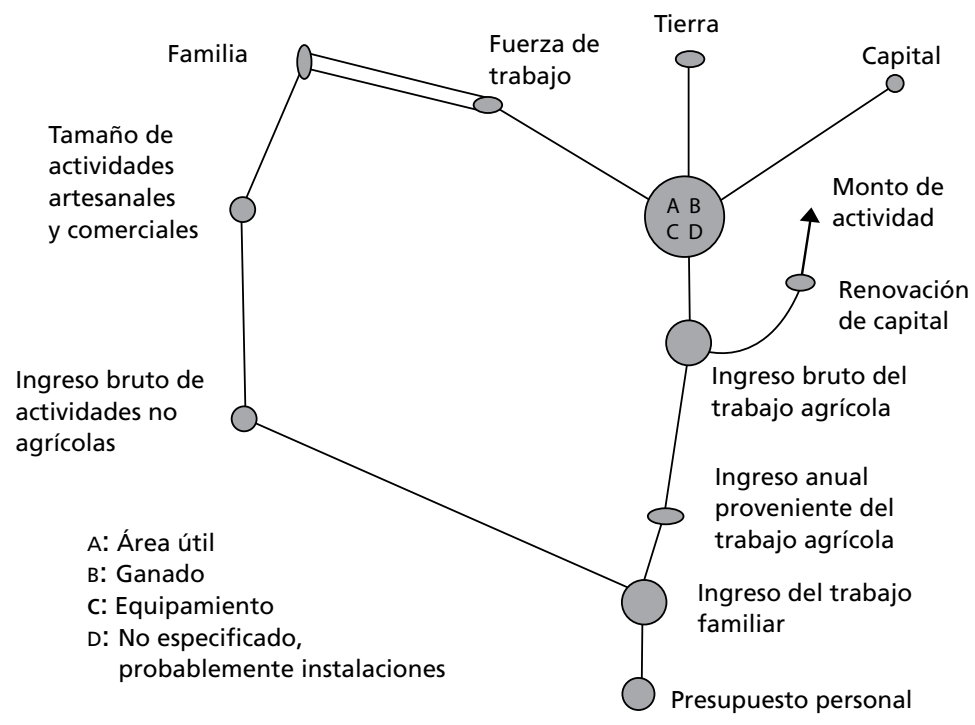

Fuente: Chayanov A. (1985, p.112).

sión del trabajo en la familia campesina entre agricultura y actividades artesanales y comercio se lleva a cabo por comparación de la situación de mercado de esas dos ramas de la economía y como la situación de ambas es fluctuante, también lo es la relación entre consumo de fuerza de trabajo en artesanías, comercio y agricultura.

\section{CONCLUSIONES}

En la historia de la teoría económica nunca se consideró que pudiera darse un cambio tan profundo en el paradigma dominante, un cambio que significara que el campesino no fuera un actor igual que cualquier otro en las sociedades industrializadas. Las preocupaciones en torno al calentamiento global, la pérdida de biodiversidad, el deterioro ecológico y la escasez de agua asociadas con dichos cambios han obligado a la reflexión del papel que desempeña el territorio rural y sus moradores para lograr la organización sustentable de la sociedad. Hay dos perspectivas propuestas para abordar estos problemas que se discuten actualmente en torno del debate sobre el futuro de la sociedad rural, ambos reclamando la etiqueta de nueva ruralidad. La primera es que la crisis del sector rural se profundiza porque las comunidades no han aceptado su nueva función, que implica una 
nueva vinculación con el sector urbano e industrial y consiste en proporcionar productos agropecuarios y servicios ambientales, así como aceptar la aportación pasiva de sus ecosistemas como sumideros de carbono.

Una segunda perspectiva parte de la existencia de un creciente número de casos de éxito en proyectos productivos de comunidades rurales que se caracterizan por combinar el manejo sustentable de recursos naturales y el bienestar de sus miembros; son capaces de generar excedentes bajo formas organizativas propias. La primera perspectiva, ortodoxa, propone una redistribución global de las capacidades productivas según los factores productivos y los impactos del cambio climático, mientras que la segunda surge de una postura heterodoxa que privilegia estas formas alternas productivas, su racionalidad y su alcance para construir nuevas propuestas de modelos económicos.

La relación entre el régimen de propiedad-racionalidad-sustentabilidad que postulan las teorías de la economía ecológica y neoinstitucional es útil para contrarrestar una de las afirmaciones que derivan de la perspectiva de racionalidad única del sistema capitalista, ya que demuestra que el régimen de propiedad colectivo no es sinónimo de degradación ambiental. Sin embargo, todavía estamos lejos de poder dar una explicación a la dinámica del territorio rural; es decisivo explicar no sólo la existencia de las racionalidades alternas, sino también examinar el porqué existe una relación estrecha entre el cuidado del ambiente y la capacidad de mejorar las condiciones de vida para la población campesina.

La EE ha venido a desempolvar una antigua propuesta de la economía campesina, la de Chayanov, para quien el progreso de Rusia dependía del fortalecimiento de la UEC. Las razones de ambas perspectivas teóricas por supuesto son diferentes: para la EE esto es decisivo por varias razones: la primera tiene que ver con la eficiencia ecológica de la producción campesina; la segunda, con los desafíos a los que el cambio climático nos enfrenta, y las respuestas a estos desafíos que ofrece el sector agrícola. La inseguridad alimentaria derivada de las variaciones en el clima, según el Panel Intergubernamental sobre el Cambio Climático (Rodríguez, 2008) afectará de forma diferenciada a los países; los países más perjudicados en términos de productividad y rendimiento por hectárea son los latinoamericanos. Al igual que sucedió en el pasado, las repercusiones en el ambiente de las formas productivas del sistema económico han vuelto a realzar al sistema campesino como una vía de producción ecológicamente sustentable. La razón de que el impacto ecológico de estas sociedades sea menor al de la capitalista radica, según consenso de los estudios del tema, es que estos grupos humanos poseen una racionalidad distinta a la capitalista, que los impulsa a ac- 
tuar bajo lógicas que no privilegian la generación de beneficios o ganancias personales únicamente.

La economía neoinstitucional y la experimental han aportado elementos importantes en la discusión sobre la existencia de una sola racionalidad: la del homo economicus. Sin embargo, no constituyen en sí mismos cuerpos teóricos que expliquen las distintas racionalidades y tampoco proporcionan una explicación integral de la dinámica de funcionamiento de una sociedad no capitalista.

En este documento se destacan algunos hechos teóricos fundamentales:

1) Existen sociedades que conviven con el sistema capitalista y que, a pesar de ello, mantienen estructuras sociales y económicas no capitalistas; este hecho puede explicar la diferencia que existe en el manejo de los recursos naturales por parte de los campesinos respecto del sistema capitalista.

2) Las racionalidades alternas identificadas no se organizan en torno del único fin de conseguir el máximo beneficio; no existe la supremacía de la esfera económica sobre la social.

3) No existe una teoría que explique de forma integral la dinámica social de la economía campesina en la actualidad.

La nueva ruralidad, en su perspectiva heterodoxa, puede proporcionar elementos para avanzar en la construcción teórica y proporciona elementos para identificar las transformaciones en el sector rural derivadas de las transformaciones estructurales del sistema económico. Su relevancia en términos de los acontecimientos internacionales actuales es enorme y desafortunadamente nuestros avances teóricos son insuficientes. Ahondar en estos temas nos podría permitir desarrollar estrategias para enfrentar con mayor eficacia la creciente pobreza y marginación de la población rural y las crisis ambientales que amenazan a la humanidad. 


\section{REFERENCIAS BIBLIOGRÁFICAS}

Barkin, D. (1998), Riqueza, pobreza y desarrollo sustentable, México, Jus-Centro Lindavista. Disponible en: http://anea.org.mx/docs/Barkin-Sostenibilidad.pdf

- (2001), "Superando el paradigma neoliberal: el desarrollo popular sustentable", en N. Giarraca (comp.), Una nueva ruralidad en América Latina. Buenos Aires, CLACSO, pp. 81-99. Disponible en: http://www.clacso.org/libros/rural/rural.html

— y M. Rosas (2006), “¿Es posible un modelo alterno de generación de excedentes?: La nueva economía indígena-campesina", Actas Latinoamericanas de Varsovia, Núm. 29, pp. 97-110.

Bonnal, P., P. M. Bosc, J. M. Díaz y B. Losch (2003), "Multifuncionalidad de la agricultura y nueva ruralidad ¿Reestructuración de las políticas públicas a la hora de la globalización?". Ponencia presentada en el seminario internacional "El Mundo Rural: Transformaciones y Perspectivas a la Luz de la Nueva Ruralidad", Bogotá, Universidad Javeriana, CLACSO, REDCAPA, octubre. Disponible en: http:// www.era-mx.org/biblio/politica/NuevaRuralidad.pdf

Borras Jr., S. (2009), "Agrarian change and peasant studies: changes, continuities, and challenges: An introduction", Journal of Peasant Studies, Vol. 36(1), pp. 5-31.

Bray, D., y L. Merino (2004), La experiencia de las comunidades forestales en México. Veinticinco años de silvicultura y construcción de empresas forestales comunitarias, México, Semarnat, INE, Consejo Civil Mexicano para la Silvicultura Sostenible, A.C. y Fundación Ford.

Calva, J. L. (1989), Los campesinos y su devenir en las economías de mercado, México, Siglo XXI Editores.

Capel, H. (2003), "El drama de los bienes comunes. La necesidad de un programa de investigación”, Revista Bibliográfica de Geografía y Ciencias Sociales, Vol. VIII, Núm. 458, Universidad de Barcelona. http://www.ub.es/geocrit/b3w-458.htm

Carpintero, O. (2006), La bioeconomía de Georgescu-Roegen, Barcelona, Montesinos.

Carrere, R. (coord.) (2003), Represas; la lucha contra los modernos dinosaurios, Montevideo, Movimiento Mundial por los Bosques Tropicales.

Cartón de Grammont, H. (2004), "La nueva ruralidad en América Latina", Revista Mexicana de Sociología, año 66 (Núm. Especial), pp. 279-300.

Chayanov, A. (1985), La organización de la Unidad económica campesina, Barcelona, Nueva Alianza.

Cortés, F., y O. Cuéllar (1986), "Lenin y Chayanov, dos enfoques no contradictorios", Revista Nueva Antropología, Vol. IX (31), pp. 63-102. 
Erickson, J., y J. Gowdy (comps.) (2007), Frontiers in ecological economic theory and application, Northampton, Ma, Edward Elgar Publishing.

Fine, B. (2009), Theories of Social Capital: Researchers Behaving Badly, Londres, Pluto.

Fisher, B, y J. Erickson (2007), "Growth and equity: dismantling the Kaldor-KuznetsSolow consensus", en Erickson y Gowdy, op. cit, pp 53-71.

Fuente Carrasco, M. E. (2009), "Nueva ruralidad comunitaria y sustentabilidad: Contribuciones al campo emergente de la economía ecológica", Revista Iberoamericana de Economía Ecológica, Núm. 13, pp. 55-69. Disponible en: http://www. redibec.org/IVO/rev13_04.pdf

Georgescu-Roegen, N. (1960), “Teoría económica y economía agraria”, El Trimestre Económico, XXXIV, 1967, pp. 589-638.

__ (1971), La ley de la entropía y el proceso económico 1996, Madrid, Fundación Argentaria/Visor.

Gomero Osorio, L., y H. Velásquez Alcantara (2003), "Evaluación de la sustentabilidad del sistema de algodón orgánico en la zona de trópico húmedo del Perú", LEISA Revista de Agroecología, Vol. 19, edición especial: Ocho estudios de caso, abril. http://latinoamerica.leisa.info/index.php?url=getblob.php\&o_id=84632\&a_ id $=211 \&$ a_seq $=1$

Gudynas, E. (2005), “Geografías fragmentadas: sitios globalizados, áreas relegadas”, Revista del Sur, Núm. 160. http://www.redtercermundo.org.uy/revista_del_sur/ texto_completo.php?id $=2815$

Henrich, J., R. Boyd, S. Bowles, C. Camerer, E. Fehr, y H. Gintis. (comps.) (2004), Foundations of human sociality. Economic Experiments and Ethnographic Evidence from Fifteen Small-Scale Societies, Oxford University Press.

Hilliard, T., M. Tattersfield, A. Rozum, y S. Kunickis (2006), "Iniciativa trinacional sobre la calidad del agua y la agricultura, tecnologías actuales: Experiencias compartidas", Agriculture and Agri-Food Canada, Prairie Farm Rehabilitation Administration, Secretaria de Medio Ambiente y Recursos Naturales, Departamento de Agricultura, Cooperative State Research, Education, and Extension Service y U.S. Department of Agriculture, Natural Resources Conservation Service, Disponible en: http://www.iisd.org/pdf/2006/natres_tri_nat_case_studies_es.pdf

Leff, E. (2004), Racionalidad ambiental la reapropiación social de la naturaleza, México, Siglo XXI Editores.

López, R. (2007), “El empoderamiento del manejo forestal comunitario en Oaxaca. La unión de comunidades forestales y ejidos de Oaxaca 1985-1996”, en D. Bray, L. Merino Pérez y D. Barry (comps.), Los bosques comunitarios de México. Manejo sustentable de paisajes forestales, México, Instituto Nacional de Ecología, 
Secretaría de Medio Ambiente y Recursos Naturales. http:/www2.ine.gob.mx/ publicaciones/consultaPublicacion.html?id_pub=532

Mantilla, J. (2005), "Cultivo ecológico de plantas medicinales y aromáticas: ampliando las perspectivas económicas en los Andes", LEISA. Revista de Agroecología, Núm. 21(2) http://www.leisa-al.org.pe/anteriores/212_pdf/33.pdf

Martínez Alier, J., y K. Schlüpmann (1991), La ecología y la economía, México, FCE. (1992), De la economía ecológica al ecologismo popular, Barcelona, Icaria.

(1994), El ecologismo de los pobres. Conflictos ambientales y lenguajes de valoración, Barcelona, Icaria.

— y J. Roca (2001), Economía ecológica y política ambiental, México, Fondo de Cultura Económica.

Marx, K. (1857), Contribución a la crítica de la economía politica, México, Siglo XXI Editores 2003.

Murga, O. (2003), "Mujeres dirigiendo el cambio rural: Comité y grupo femenino colonia el Quetzal, Tacaná, Guatemala", LEISA. Revista de Agroecología, Vol. 18(4), pp. 5-6. http://latinoamerica.leisa.info/index.php?url=getblob.php\&o_id=67131\&a_ $\mathrm{id}=211 \& \mathrm{a} \_$seq $=0$

Naredo, J. M. (1994), "Fundamentos de la economía ecológica”, en F., Aguilera Klink y V. Alcántara (comps.), De la economía ambiental a la economía ecológica, Barcelona, Icaria.

Ostrom, E. (1990), El gobierno de los bienes comunes. La evolución de las instituciones de la acción colecta, México, Fondo de Cultura Económica, 2000.

Reijntjes, C. (2009) “Los pequeños agricultores: la clave para conservar la biodiversidad", LEISA. Revista de Agroecología, Vol. 25(1). http://latinoamerica.leisa.info/ index.php?url=getblob.php\&o_id=230758\&a_id=211\&a_seq $=0$

Rodríguez Herrera, A., y A. Ugarte (2008), Claves de la innovación social en América Latina y el Caribe, Libros de la CEPAL 101, Santiago de Chile, CEPAL, disponible en: http://www.cepal.org/publicaciones/xml/2/34682/Claves_de_innovacion _social.pdf

Saubot, P. (2005), Introducción general al medio ambiente y ecología, Rosario, Argentina. http://www.elestanque.com/articulos/medio_ambiente.html (consultado 9/2/07).

Tapella, E. (2004), "Reformas estructurales en Argentina y su impacto sobre la pequeña agricultura. ¿Nuevas ruralidades, nuevas políticas?”, Estudios Sociológicos, Núm. 66, disponible en web: http://revistas.colmex.mx/resultados_busqueda. jsp?tema $=19441$

Toledo, V. M. (1992), "What is ethnoecology? Origins, scope, and implications of a rising discipline", Etnoecologica, Vol. 1, pp. 5-21. 